\title{
Training the Next Generation of Neurosurgeons in Developing Countries - Mission Possible
}
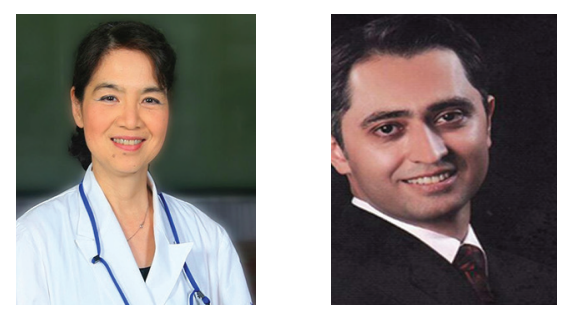

Yoko Kato ${ }^{1}$, Mohsen Nouri ${ }^{*}$

${ }^{1}$ Professor and Chair, Department of Neurosurgery, Banbuntane Hotokukai Hospital, Fujita Health University, Aichi, Japan

2 Neurosurgeon, Department of Neurosurgery, Gundishapour Academy of Neuroscience, Ahvaz, Iran

*Corresponding Author Address: Department of Neurosurgery, Gundishapour Academy of Neuroscience, Ahvaz, Iran.

Tel: +989375325455. Email: nouri@gan-ac.ir

Article Type: Editorial

Received: May 28, 2017, Last Revised: June 12, 2017, Accepted: June 21, 2017, Published: June 30, 2017

Please cite this paper as: Kato Y, Nouri M. Training the Next Generation of Neurosurgeons in Developing Countries - Mission Possible. Iran J Neurosurg. 2017;3(1):6-7

One of the main concerns in different countries is training young neurosurgeons to treat patients. Each country is dealing with this issue with a certain strategy considering its goals. Training physicians is far different from many other fields, as it cannot be accomplished in the library or by reading books. This fact becomes even more notable when it comes to the neurosurgery which requires meticulous surgical skills and knowledge. Training a capable neurosurgeon starts with the process of accepting, develops with residency education, and is solidified by a post-graduation training (e.g. fellowships) which will be reviewed briefly here.

The traditional "concours" is the elementary way to select a resident. Relying solely on a single day exam with its own limitations - might be the easiest but definitely not the best way to select a neurosurgery candidate. More advanced systems, interview individuals based on their academic background, exam results, personal communications, and recommendations. This allows the program directors to know the candidates in person, their goals, personalities, and ambitions. However, this requires organization and a dedicated system whose infra-structures may not be available in many countries.

Education of neurosurgery residents is also variable among different countries and different centers in the same country. Cadaver labs are inseparable part of neurosurgical education in the US and are used for learning neurosurgical anatomy, skull base approaches, and spinal instrumentation. Many centers dedicate one entire year for research to educate young residents in different aspects of clinical and basic neurosurgical research. This is of paramount importance to train a generation of neurosurgeons capable of advancing the field of neurosurgery and world leaders of the future. Organizing local, national, and international workshops (e.g. presentations, or hands-on courses) and invited speakers are an important part of education to expose residents to a variety of methods and philosophies in practice.

Though general neurosurgeons may still be needed in under- or developing countries, subspecialty training is becoming more and more necessary in well-developed regions. This may be in the form of clinical hands-on fellowships in one to two years or working as a junior neurosurgeon under experienced professors to gain exposure over years. This 
is what makes the quality of treatment different among the countries. As high quality fellowships in under developed countries are not available, neurosurgeons should look for such training in developed countries. Unfortunately, pursuing a formal neurosurgical training is very difficult for the physicians from developing countries. Official hands-on training requires many exams and is very competitive, so most such trainings are limited to observerships which may not be enough for technically demanding surgeries (e.g. skull base or vascular lesions). Unfortunately, this may increase the gap between developed and developing countries in terms of sophisticated surgical techniques. Even under-developed countries have general neurosurgeons and residency programs training neurosurgeons. These trained neurosurgeons are capable of performing general approaches which consist majority of daily practices but when it comes to complicated cases, subspecialty training is required to address them appropriately and as most these countries lack subspecialty trained neurosurgeons, these patients are usually undertreated.

By reviewing three steps in training neurosurgeons, we believe that developing countries should focus on the third step (i.e. post residency training) to improve the quality of care and educate a generation of neurosurgeons capable of performing sophisticated surgeries. The first (i.e. selecting residents) and the second steps (i.e. residency training) are also important, but even the best quality of each does not guarantee high quality treatment, because such a system can only train well educated general neurosurgeons at its best. However, these two steps are definitely necessary to achieve the aim of the third step. In short term, international workshops, visiting fellowships, and educational materials (e.g. books, videos, atlases, websites, etc) seem the only ways to achieve this goal. Yet, higher quality training (e.g. hands on fellowships) should be considered to deliver top-notch knowledge to the developing countries in the future. 